\title{
Evaluation of the neonatal outcomes of the kangaroo mother method in Brazil
}

\author{
Avaliação dos resultados neonatais do método canguru no Brasil \\ Fernando Lamy Filho', Antônio Augusto Moura da Silva ${ }^{2}$, Zeni Carvalho Lamy ${ }^{3}$, \\ Maria Auxiliadora Sousa Mendes Gomes ${ }^{4}$, Maria Elizabeth Lopes Moreira ${ }^{4}$, \\ Grupo de Avaliação do Método Canguru ${ }^{5}$, Rede Brasileira de Pesquisas Neonatais ${ }^{6}$
}

\section{Resumo}

Objetivo: Avaliar os resultados do método canguru no Brasil.

Métodos: Estudo de coorte prospectivo comparando 16 unidades que possuíam ou não a segunda fase do método canguru: oito eram centros nacionais de referência para o método canguru (grupo estudo), e oito faziam parte da Rede Brasileira de Pesquisas Neonatais (grupo controle). Foram incluídos 985 recém-nascidos pesando entre 500 e $1.749 \mathrm{~g}$. Na análise multivariada, utilizou-se a regressão linear múltipla e a regressão de Poisson com ajuste robusto.

Resultados: Na análise ajustada (para peso de nascimento, idade gestacional, Score for Neonatal Acute Physiology Perinatal Extension II, Neonatal Therapeutic Intervention Scoring System, idade e escolaridade maternas), o tempo médio de internação $(p=0,14)$ e intercorrências clínicas na unidade intermediária ou canguru foram iguais entre os grupos. Peso $(p=0,012)$, comprimento $(p=0,039)$ e perímetro cefálico $(p=0,006)$ com 36 semanas de idade gestacional corrigida foram menores nas unidades canguru. As unidades canguru tiveram desempenho superior em relação ao aleitamento materno exclusivo na alta $(69,2$ versus $23,8 \%, p=0,022)$.

Conclusão: As evidências sugerem que a estratégia de humanização adotada pelo Ministério da Saúde é uma alternativa segura ao tratamento convencional e uma boa estratégia para a promoção do aleitamento materno.

J Pediatr (Rio J). 2008;84(5):428-435: Método canguru, baixo peso ao nascer, humanização.

\author{
Abstract \\ Objective: To evaluate the results of the kangaroo mother \\ method in Brazil.
}

Methods: A prospective cohort study comparing 16 units that have or do not have the second phase of the kangaroo mother method: eight were national centers of excellence for the kangaroo mother method (study group) and eight were part of the Brazilian Neonatal Research Network (control group). A total of 985 newborn infants with birth weights of 500 to $1,749 \mathrm{~g}$ were enrolled. Multivariate analyses employed multiple linear regression and Poisson regression with robust adjustment.

Results: The adjusted analysis (controlled for birth weight, gestational age, Score for Neonatal Acute Physiology Perinatal Extension II, Neonatal Therapeutic Intervention Scoring System, and maternal age and educational level) demonstrated that mean length of hospital stay $(p=0.14)$ and intercurrent clinical conditions in the intermediate or kangaroo unit were equal for both groups. Weight ( $p$ $=0.012)$, length $(p=0.039)$ and head circumference $(p=0.006)$ at 36 weeks' corrected gestational age were all lower at the kangaroo units. The kangaroo units exhibited superior performance in relation to exclusive breastfeeding at discharge ( 69.2 vs. $23.8 \%, p=0.022)$.

Conclusions: The evidence suggests that the humanization strategy adopted by the Brazilian Ministry of Health is a safe alternative to conventional treatment and a good strategy for promoting breastfeeding.

J Pediatr (Rio J). 2008;84(5):428-435: Kangaroo mother care, low birth weight, humanization.

1. Doutor. Professor adjunto, Departamento de Medicina III, Universidade Federal do Maranhão (UFMA), São Luís, MA.

2. Doutor. Professor associado, Departamento de Saúde Pública, UFMA, São Luís, MA.

3. Doutora. Professora adjunta, Departamento de Saúde Pública, UFMA, São Luís, MA.

4. Doutora. Pesquisadora, Instituto Fernandes Figueira, Fundação Oswaldo Cruz (Fiocruz), Rio de Janeiro, RJ.

5. Grupo de Avaliação do Método Canguru: Geisy Maria Souza Lima (Instituto Materno-Infantil de Pernambuco - IMIP, PE), Susane Oliveira de Menezes, Arnaldo Costa Bueno (Secretaria Municipal da Saúde do Rio de Janeiro, RJ), Olga Penalva Vieira da Silva (Hospital de Itapecerica da Serra, SP), Sílvia Helena Cavalcante de Souza Godoy (Hospital Universitário da Universidade Federal do Maranhão, MA).

6. Rede Brasileira de Pesquisas Neonatais: José Maria de Andrade Lopes, Carla Nasser Patrocínio, Ana Beatriz de Souza Machado (Instituto Fernandes Figueira, Fiocruz, RJ), Ruth Guinsburg, Maria Fernanda Branco de Almeida, Ana Lúcia Goulart (Hospital São Paulo, UNIFESP, SP), Renato Machado Fiori (Hospital São Lucas, PUCRS, RS), Cleide Enoir Petean Trindade (UNESP-Botucatu, SP), Cléa Rodrigues Leone (USP, SP), Lilian dos S. R. Sadeck (Instituto da Criança, USP, SP), Renato Soibelmann Procianoy, Rita C. Silveira (Hospital de Clínicas de Porto Alegre, UFRGS, RS), Francisco Eulógio Martinez (Hospital de Clínicas da Faculdade de Medicina de Ribeirão Preto, USP, RS), Sérgio Tadeu Martins Marba, Gisele Marafon Lopes de Lima (Centro de Atenção Integral à Saúde da Mulher, UNICAMP, SP).

Pesquisa desenvolvida por iniciativa da área técnica de Saúde da Criança e Aleitamento Materno do Ministério da Saúde, financiada pelo Ministério da Saúde e pela Organização Pan-Americana da Saúde.

Não foram declarados conflitos de interesse associados à publicação deste artigo.

Como citar este artigo: Lamy Filho F, Silva AA, Lamy ZC, Gomes MA, Moreira ME; Grupo de Avaliação do Método Canguru; Rede Brasileira de Pesquisas Neonatais. Evaluation of the neonatal outcomes of the kangaroo mother method in Brazil. J Pediatr (Rio J). 2008;84(5):428-435.

Artigo submetido em 06.11.07, aceito em 05.06.08.

doi:10.2223/JPED. 1821 


\section{Introdução}

O cuidado integral ao bebê e sua família tem se tornado uma das boas práticas observadas durante a internação em unidade de terapia intensiva neonatal (UTIN) ${ }^{1-4}$. Desde 1999, o Ministério da Saúde vem implantando a política de atenção humanizada ao recém-nascido de muito baixo peso (método canguru $)^{5}$, uma proposta de humanização da assistência neonatal baseada em quatro fundamentos básicos: acolhimento ao bebê e sua família, respeito às singularidades, promoção do contato pele a pele (posição canguru) e o envolvimento da mãe nos cuidados com o filho ${ }^{6}$.

O método canguru foi originalmente desenvolvido por Rey \& Martinez ${ }^{7}$ em 1979 na cidade de Bogotá. O recém-nascido prematuro era colocado em contato pele a pele, entre os seios maternos, após estabilização clínica. Sua utilização se justificava pela falta de incubadoras e o alto índice de mortalidade nas maternidades colombianas ${ }^{7}$.

A partir de 1984, o método passou a ser amplamente divulgado pelo Fundo das Nações Unidas para a Infância (UNICEF). Vários autores afirmavam que bebês submetidos ao método canguru apresentavam menor tempo de internação, oxigenação adequada, aumento e estabilização da temperatura corporal, menos episódios de apnéia e pouco choro ${ }^{8,9}$. Além disso, as mães amamentavam mais e sentiam-se seguras em monitorar a saúde de seus bebês.

Experiências com o método mostraram redução dos riscos de infecção hospitalar às 41 semanas de idade gestacional (IG) corrigida e redução de doença grave, principalmente do aparelho respiratório 6 meses pós-alta. Observou-se também a redução do risco de insatisfação da mãe, de falha da amamentação exclusiva na alta e a melhora do ganho ponderal durante a internação ${ }^{10-19}$.

No entanto, as definições de método ou assistência canguru utilizadas nesses trabalhos não são uniformes, variando conforme a instituição. A maioria refere-se apenas ao uso do contato pele a pele em algum momento da internação do bebê. No Brasil, o método canguru consiste em um esforço de humanização da assistência que envolve três etapas: a internação na UTIN, na unidade canguru e o acompanhamento no ambulatório de seguimento após a alta hospitalar até o peso de $2.500 \mathrm{~g}$. Em todas as etapas, o contato pele a pele e o aleitamento materno são incentivados. Além disso, na segunda etapa, a mãe permanece, diuturnamente, assumindo os cuidados do seu filho progressiva e continuamente até a alta hospitalar ${ }^{5}$.

Verificou-se, então, a necessidade de um estudo que avaliasse especificamente a experiência brasileira com o método canguru, uma vez que não existem evidências científicas suficientes na literatura sobre o impacto deste tipo de manejo em indicadores de evolução neonatal ${ }^{20}$.

O presente trabalho visou avaliar os resultados neonatais da política de atenção humanizada ao recém-nascido de baixo peso (método canguru), na qual o contato pele a pele é uma parte do cuidado. Especificamente, buscou-se descrever as características perinatais dos recém-nascidos incluídos no estudo, comparar tempo de permanência na unidade intermediária, retorno para a UTIN, crescimento e indicadores clínicos de resultado do cuidado neonatal durante a internação nas unidades de cuidados intermediários convencionais e unidades canguru (segunda etapa do método).

\section{Métodos}

Estudo de coorte, prospectivo, de recém-nascidos internados em unidades neonatais públicas brasileiras acompanhados do nascimento até a alta. Considerou-se unidade neonatal o setor de internação envolvendo UTIN, unidade intermediária e unidade canguru. Apesar de o estudo ter sido realizado durante a internação em unidade intermediária ou canguru, todos os serviços ofereciam terapia intensiva. Foram consideradas unidades estudo aquelas que contavam com unidade canguru, ou seja, dispunham de leitos destinados à internação conjunta mãe-bebê, aplicavam as três etapas do método canguru e eram centros de referência para o Ministério da Saúde. As unidades controle eram aquelas que utilizavam a unidade intermediária convencional, apresentavam bons resultados neonatais e ações de humanização, mas que não praticavam a segunda etapa do método canguru - internação conjunta mãe-bebê - todas pertencentes à rede brasileira de neonatologia.

Participaram do estudo 16 unidades neonatais. Oito eram centros de referência do Ministério da Saúde para o método canguru, e oito eram integrantes da Rede Brasileira de Pesquisas Neonatais, somando cerca de 3.000 internações/ano de menores de $1.750 \mathrm{~g}$. Todas tiveram seu nível de complexidade classificado através da proposta da Vermont Oxford Network no Vermont Oxford Annual Meeting, 2002. As unidades controle (cuidado convencional) foram: Instituto Fernandes Figueira/Fundação Oswaldo Cruz (Fiocruz), Rio de Janeiro, RJ; Hospital de Clínicas de Porto Alegre/Universidade Federal do Rio Grande do Sul (HCPA/UFRGS) e Hospital São Lucas, Pontifícia Universidade Católica do Rio Grande do Sul (PUCRS), Porto Alegre, RS; Hospital São Paulo/Universidade Federal de São Paulo (UNIFESP) e Instituto da Criança/ Universidade de São Paulo (USP), São Paulo, SP; Faculdade de Medicina de Botucatu, Universidade Estadual de São Paulo (UNESP), Botucatu, SP; Centro de Atenção Integral à Saúde da Mulher (Unicamp), Campinas, SP e Hospital de Clínicas da Faculdade de Medicina de Ribeirão Preto(FMRP-USP), Ribeirão Preto, SP. As unidades de estudo (cuidados canguru) foram: Hospital Universitário Unidade Materno-Infantil (UFMA), Instituto Materno-Infantil de Pernambuco (IMIP), Hospital Geral de Itapecerica da Serra (SP) e cinco Maternidades Municipais do Rio de Janeiro: Alexander Fleming, Oswaldo Nazareth, Carmela Dutra, Herculano Pinheiro e Instituto da Mulher Fernando de Magalhães.

\section{População do estudo}

Foram incluídos recém-nascidos com peso ao nascer entre 500 e $1.749 \mathrm{~g}$, pois a partir de $1.750 \mathrm{~g}$, em alguns serviços, 
muitos bebês já são encaminhados diretamente aos alojamentos conjuntos logo após o nascimento. Foram excluídos recém-nascidos com malformações maiores, portadores de infecção congênita do grupo TORCHS (toxoplasmose, rubéola, citomegalovírus, herpes simples e sífilis), cromossomopatias, anomalias metabólicas, recém-nascidos com encefalopatia perinatal grave ou com enterocolite necrosante que requereram cirurgia.

Os critérios para a transferência da UTIN para a unidade canguru foram estabilidade clínica, peso superior a $1.250 \mathrm{~g}$, nutrição enteral plena (por sonda orogástrica ou sucção) e estar respirando em ar ambiente sem relato de apnéias que tenham necessitado de reanimação com oxigênio e pressão positiva nos últimos 5 dias. No grupo controle, os critérios de admissão e alta variaram nas diversas unidades.

\section{Coleta de dados}

Os dados foram coletados no período de março de 2004 a março de 2005. A supervisão e o controle de qualidade das informações foram realizados por coordenadores do trabalho de campo, responsáveis pelo treinamento da equipe local a partir de manual de instruções.

As variáveis-resposta relativas à internação na unidade intermediária ou unidade canguru foram: tempo de permanência; média de ganho de peso em g/kg/dia; peso, comprimento e perímetro cefálico $(\mathrm{PC})$ às 36 semanas de IG corrigida se o bebê continuasse internado; além da ocorrência de distermia, apnéia, infecção, retorno para a UTIN e aleitamento exclusivo na alta.

Foram consideradas variáveis de confundimento: escolaridade e idade maternas, número de filhos vivos, situação conjugal, peso de nascimento, IG ao nascimento, escores de gravidade inicial (Score for Neonatal Acute Physiology Perinatal Extension II - SNAPPE II $)^{21}$ e de intensidade de uso de tecnologia e utilização de recursos (Neonatal Therapeutic Intervention Scoring System - NTISS) ${ }^{22}$, tempo de permanência na UTIN e peso e IG corrigida na admissão na unidade intermediária ou unidade canguru.

\section{Cálculo do tamanho da amostra}

Para um tempo de internação de 26 dias para bebês com peso entre 500 e $1.749 \mathrm{~g}$, com desvio padrão de 24 dias, um tamanho de amostra total de 756 permitiria detectar diferenças na média do tempo de internação de 4 dias (diferença relevante), assumindo-se poder de $90 \%$ e probabilidade de erro do tipo I de $5 \%$.

Para uma média de ganho de peso medida em g/ $\mathrm{kg} / \mathrm{dia}$ de $14 \mathrm{~g}$ para bebês com peso de nascimento menor do que $1.500 \mathrm{~g}$, e desvio padrão de $7 \mathrm{~g}$, um tamanho de amostra total de 257 permitiria detectar diferenças na média do ganho de peso de $2 \mathrm{~g} / \mathrm{kg} /$ dia (diferença relevante), assumindo-se poder de $90 \%$ e probabilidade de erro do tipo I de $5 \%$.

Para variáveis categóricas, 804 bebês em cada grupo permitiriam identificar risco relativo de 1,7 para variáveis cuja prevalência no grupo não exposto esteja em torno de $10 \%$, com um poder de $80 \%$ e uma probabilidade de erro tipo I de $5 \%$. Um tamanho de amostra total de 948 permite detectar risco relativo de 2 para variáveis cuja prevalência seja de $5 \%$ com poder de $80 \%$ e alfa de $5 \%$. A amostra final incluiu 985 recém-nascidos nos dois grupos.

\section{Análise estatística}

Na descrição das variáveis quantitativas, usou-se média e desvio padrão. A comparação das médias entre as unidades foi feita pelo teste $t$ de Student. As variáveis categóricas foram descritas usando-se percentuais. A diferença das proporções entre as unidades foi verificada pelo teste do qui-quadrado. $\mathrm{Na}$ análise multivariada, para variáveis quantitativas usou-se a regressão linear múltipla e, para variáveis binárias, a regressão de Poisson com ajuste robusto, devido ao fato de muitas variáveis-resposta não serem eventos raros. O uso da regressão logística poderia levar a uma superestimativa dos efei$\operatorname{tos}^{23}$. Todas as análises foram ajustadas para o efeito cluster, considerando-se que observações de indivíduos nascidos na mesma unidade não são independentes. No caso de variáveisresposta quantitativas, como muitas delas tinham assimetria e/ou curtose e em alguns modelos a análise de resíduos mostrou problemas com os pressupostos, todos os modelos foram submetidos a bootstrapping para verificar se a quebra dos pressupostos poderia levar a resultados diferentes. Como os resultados foram semelhantes em modelos com regressão linear e por bootstrapping, apresentaram-se os resultados da regressão linear obtida pelo método dos mínimos quadrados, pois com o programa disponível (Stata versão 8.0) não foi possível obter estimativas por bootstrapping levando-se em conta o efeito cluster. O projeto foi aprovado pelos Comitês de Ética em Pesquisa de cada uma das unidades envolvidas. Em todas as unidades, os representantes assinaram termo concordando com a pesquisa, e os pais assinaram termo de consentimento livre e esclarecido para inclusão de cada recémnascido no estudo.

\section{Resultados}

Os recém-nascidos das unidades canguru (estudo) apresentaram peso e IG maiores que aqueles das unidades intermediárias (controle). Índices de SNAPPE II, NTISS, idade e escolaridade maternas foram maiores nas unidades intermediárias. Não houve diferença em relação ao sexo e situação conjugal materna. Mães com maior número de filhos e maior tempo de permanência na UTIN predominaram no grupo estudo, com significância marginal (valor de p entre 0,10 e 0,05) (Tabela 1).

Na análise não ajustada, os recém-nascidos das unidades canguru tiveram tempo de permanência menor $(18,9$ versus 24,1 dias) que os internados nas unidades convencionais. A diferença foi de 5,2 dias a menos para o grupo canguru, sendo marginalmente significante $(p=0,067)$. Após o ajuste, essa diferença diminuiu para $-4,3$ dias e não foi significante $(p=$ 0,140) (Tabela 2).

Os recém-nascidos das unidades controle tiveram média de ganho ponderal ligeiramente superior às das unidades 
Tabela 1 - Características dos recém-nascidos admitidos no estudo, internados em unidades canguru ou convencional (Brasil, 2005)

\begin{tabular}{|c|c|c|c|c|c|}
\hline \multirow[b]{2}{*}{ Variáveis } & \multicolumn{2}{|c|}{ Unidades controle } & \multicolumn{2}{|c|}{ Unidades canguru } & \multirow[b]{2}{*}{$\mathbf{p}$} \\
\hline & $\mathbf{n}$ & Média (DP) & $\mathbf{n}$ & Média (DP) & \\
\hline Peso ao nascer & 366 & $1.313(302)$ & 621 & $1.399(255)$ & $<0,001$ \\
\hline IG & 362 & $31,7(2,8)$ & 618 & $32,4(2,3)$ & $<0,001$ \\
\hline SNAPPE II & 351 & $12,7(14,2)$ & 615 & $11,0(12,2)$ & 0,045 \\
\hline NTISS & 322 & $13,0(6,8)$ & 615 & $10,4(4,2)$ & $<0,001$ \\
\hline Idade materna & 357 & $26,6(7,3)$ & 619 & $24,8(6,9)$ & $<0,001$ \\
\hline Número de filhos & 355 & $1,8(1,7)$ & 612 & $2,0(1,6)$ & 0,076 \\
\hline Tempo de UTI & 354 & $18,6(18,7)$ & 609 & $21,1(20,7)$ & 0,067 \\
\hline Variáveis & $\mathbf{n}$ & $\%$ & $\mathbf{n}$ & $\%$ & $\mathbf{p}$ \\
\hline Sexo & & & & & 0,175 \\
\hline Masculino & 161 & 44,2 & 300 & 48,7 & \\
\hline Feminino & 203 & 55,8 & 316 & 51,3 & \\
\hline Situação conjugal & & & & & 0,224 \\
\hline Com companheiro & 263 & 79,7 & 455 & 76,2 & \\
\hline Sem companheiro & 67 & 20,3 & 142 & 23,8 & \\
\hline Escolaridade materna & & & & & 0,002 \\
\hline Nenhuma & 10 & 3,2 & 13 & 2,2 & \\
\hline Fundamental incompleto & 96 & 31,0 & 263 & 43,5 & \\
\hline Fundamental completo & 89 & 28,7 & 164 & 27,1 & \\
\hline Ensino médio & 98 & 31,6 & 146 & 24,1 & \\
\hline Superior & 17 & 5,5 & 19 & 3,1 & \\
\hline
\end{tabular}

estudo (15,3 versus $13,2 \mathrm{~g} / \mathrm{kg} / \mathrm{dia})$, com diferença significante na análise não ajustada e não significante na análise multivariada ( $p=0,220)$ (Tabela 2$)$.

Os bebês das unidades convencionais tiveram média de peso com 36 semanas de IG corrigida significantemente maior (1.709 versus $1.552 \mathrm{~g}$ ) do que os bebês das unidades canguru nas duas análises com (valores de $p=0,013$ e 0,012) (Tabela 2).

O comprimento com 36 semanas de IG corrigida não foi significantemente diferente na análise não ajustada. Porém, na análise ajustada o comprimento com 36 semanas de IG corrigida foi maior nos bebês do grupo controle, mostrando significância estatística ( $p=0,039$ ). O PC com 36 semanas de IG corrigida foi superior no grupo controle, nas duas análises $(p=0,006)$ (Tabela 2$)$.

$\mathrm{Na}$ análise não ajustada, retinopatia da prematuridade foi mais comum em unidades convencionais (significância marginal), enquanto aleitamento materno exclusivo na alta foi mais freqüente nos bebês das unidades canguru. Não houve diferença em relação às outras variáveis. $\mathrm{Na}$ análise ajustada, somente o risco de não ter alta em amamentação exclusiva permaneceu aumentado para as crianças das unidades convencionais (Tabela 3).

\section{Discussão}

Na análise ajustada (para peso de nascimento, IG, SNAPPE II, NTISS, idade e escolaridade maternas), tempo de permanência, ganho ponderal, retorno para a UTIN e ocorrência de intercorrências clínicas foram iguais entre os grupos. Peso, comprimento e PC com 36 semanas de IG corrigida foram menores nas unidades canguru. As unidades canguru tiveram desempenho nitidamente superior quanto ao aleitamento materno exclusivo na alta. Não foram observadas diferenças significantes de morbidade e mortalidade após a saída da UTI nos dois grupos. 
Tabela 2 - Tempo de permanência e dados de crescimento durante a internação em unidade intermediária convencional ou canguru (Brasil, 2005)

\begin{tabular}{|c|c|c|c|c|c|c|c|c|c|c|}
\hline \multirow[b]{2}{*}{ Variáveis } & \multicolumn{2}{|c|}{$\begin{array}{l}\text { Unidades } \\
\text { controle }\end{array}$} & \multicolumn{2}{|c|}{$\begin{array}{l}\text { Unidades } \\
\text { canguru }\end{array}$} & \multicolumn{3}{|c|}{ Análise não ajustada } & \multicolumn{3}{|c|}{ Análise ajustada* } \\
\hline & $\mathbf{n}$ & $\begin{array}{r}\text { Média } \\
\text { (DP) }\end{array}$ & $\mathbf{n}$ & $\begin{array}{l}\text { Média } \\
\text { (DP) }\end{array}$ & Coef & IC95\% & $\mathbf{p}$ & Coef & IC95\% & $\mathbf{p}$ \\
\hline $\begin{array}{l}\text { Tempo de permanência na } \\
\text { unidade intermediária } \\
\text { convencional ou canguru }\end{array}$ & 366 & $\begin{array}{c}24,1 \\
(12,8)\end{array}$ & 619 & $\begin{array}{c}18,9 \\
(11,4)\end{array}$ & $-5,2$ & $-10,9$ a 0,4 & 0,067 & $-4,3$ & $-10,2$ a 1,6 & 0,140 \\
\hline $\begin{array}{l}\text { Ganho ponderal na unidade } \\
\text { intermediária convencional ou } \\
\text { canguru (g/kg/dia) }\end{array}$ & 333 & $\begin{array}{c}15,3 \\
(11,1)\end{array}$ & 602 & $\begin{array}{c}13,2 \\
(10,2)\end{array}$ & $-2,0$ & $-3,7$ a $-0,3$ & 0,027 & $-1,2$ & $-3,3$ a 0,8 & 0,220 \\
\hline $\begin{array}{l}\text { Peso com } 36 \text { semanas de IG } \\
\text { corrigida }\end{array}$ & 302 & $\begin{array}{l}1.709 \\
(380)\end{array}$ & 534 & $\begin{array}{l}1.552 \\
(266)\end{array}$ & -156 & $-275 a-38$ & 0,013 & -191 & $-335 a-48$ & 0,012 \\
\hline $\begin{array}{l}\text { Comprimento com } 36 \text { semanas de } \\
\text { IG corrigida }\end{array}$ & 199 & $\begin{array}{l}41,8 \\
(3,0)\end{array}$ & 404 & $\begin{array}{l}41,1 \\
(2,8)\end{array}$ & $-0,7$ & $-1,6$ a 0,3 & 0,143 & $-0,9$ & $-1,8 \mathrm{a}-0,1$ & 0,039 \\
\hline $\begin{array}{l}\text { PC com } 36 \text { semanas de IG } \\
\text { corrigida }\end{array}$ & 200 & $\begin{array}{l}30,7 \\
(2,4)\end{array}$ & 465 & $\begin{array}{l}30,2 \\
(1,9)\end{array}$ & $-0,5$ & $-1,0$ a $-0,1$ & 0,040 & $-0,7$ & $-1,2 \mathrm{a}-0,2$ & 0,006 \\
\hline
\end{tabular}

Coef = coeficiente; DP = desvio padrão; IC95\% = intervalo de confiança de 95\%; IG = idade gestacional; PC = perímetro cefálico.

* Modelos ajustados para peso de nascimento, idade gestacional, SNAPPE II, NTISS, idade e escolaridade maternas e corrigidos pelo efeito cluster (unidade de nascimento). Os totais diferem para cada variável por causa de dados ignorados.

Os maiores peso, comprimento e PC com 36 semanas de IG corrigida nas unidades convencionais provavelmente refletem melhores condições de suporte nutricional durante a permanência na UTI dessas unidades, já que não houve diferença no ganho médio de peso durante a internação na unidade intermediária ou canguru. Os dados sugerem que, no grupo estudo, o aporte nutricional pode estar sendo iniciado mais tardiamente, refletindo a menor densidade tecnológica destas unidades quanto à questão nutricional e piores condições de infra-estrutura para nutrição parenteral. A literatura não estabelece evidência de maior efetividade do método canguru quanto ao ganho ponderal: Roberts et al. ${ }^{24}$ e Charpak et al. ${ }^{18}$ não encontraram diferenças, enquanto Ramanathan et al. ${ }^{13}$ e Cattaneo et al. ${ }^{25}$ observaram maior ganho de peso no grupo canguru.

Neste estudo, não houve diferença no tempo médio de permanência nos dois grupos, diferentemente do menor tempo no grupo canguru observado por Charpak et al. ${ }^{18}$ e Cattaneo et al. ${ }^{25}$. Esses dados concordam com os trabalhos de et al. ${ }^{26}$ e Roberts et al. ${ }^{24}$. Por outro lado, Ramanathan et al. ${ }^{13} \mathrm{e}$ Charpak et al. ${ }^{18}$ encontraram menor tempo de permanência nos bebês do método canguru.

Em nosso estudo, a maior diferença encontrada foi quanto ao aleitamento materno exclusivo na alta (2,34 vezes mais freqüente no grupo canguru), diferente do relatado por Sloan et al. ${ }^{27}$, que não observaram diferença entre os grupos. Roberts et al. ${ }^{24}$ encontraram tempo de amamentação igual entre os grupos canguru e convencional. Ramanathan et al. ${ }^{13}$, ao analisarem os bebês submetidos ao método canguru às 6 semanas de seguimento, encontraram o dobro de mães com amamentação exclusiva em comparação com o grupo controle.

Não houve diferença em relação a algumas intercorrências clínicas, ao contrário de Sloan et al. ${ }^{26}$, que observaram menor ocorrência de apnéia e infecções no grupo canguru, de Cattaneo et al. ${ }^{25}$, que descreveram menor risco de hipotermia, e de Kadam et al. ${ }^{26}$, que encontraram menor incidência de hipotermia, melhores níveis de saturação de oxigênio e menores freqüências respiratórias no grupo canguru. Porém, neste último estudo, não se encontrou diferença estatística entre os dois grupos quanto à ocorrência de hipertermia, sepse e apnéia.

Algumas limitações dificultaram a interpretação de alguns dados. Uma parcela de bebês pode ter atingido IG de 36 semanas corrigidas ainda dentro da UTI. Outros tiveram alta antes de completar essa IG. É provável que tenha acontecido contaminação no estudo. Em todas as unidades havia profissionais de saúde que participaram do treinamento de recursos humanos promovido pelo projeto canguru do Ministério da Saúde, e muitas das práticas preconizadas pelo programa da atenção humanizada eram praticadas nas unidades controle. Quase todas as unidades controle apresentaram práticas de contato pele a pele e muitas tinham alojamento e sala de estar 
Tabela 3 - Mortalidade e principais afecções clínicas durante a permanência em unidade convencional ou canguru (Brasil, 2005)

\begin{tabular}{|c|c|c|c|c|c|c|c|c|c|c|}
\hline \multirow[b]{2}{*}{ Variáveis } & \multicolumn{2}{|c|}{$\begin{array}{l}\text { Unidade } \\
\text { controle }\end{array}$} & \multicolumn{2}{|c|}{$\begin{array}{l}\text { Unidade } \\
\text { canguru }\end{array}$} & \multicolumn{3}{|c|}{ Análise não ajustada } & \multicolumn{3}{|c|}{ Análise ajustada* } \\
\hline & $\mathbf{n}$ & $\%$ & $\mathbf{n}$ & $\%$ & $\mathbf{R} \mathbf{R}$ & IC95\% & p & $\mathbf{R R}$ & IC95\% & $\mathbf{p}$ \\
\hline
\end{tabular}

Retorno à UTI

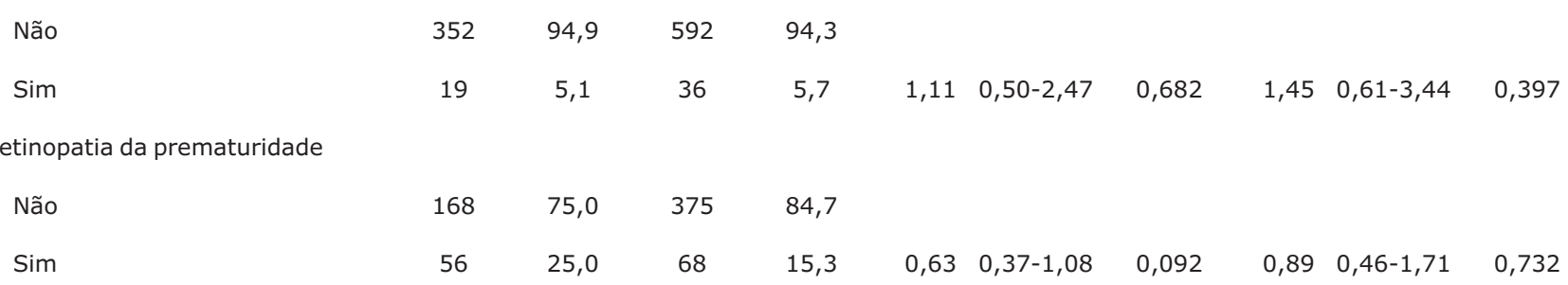

Enterocolite necrosante

\begin{tabular}{|c|c|c|c|c|c|c|c|c|c|c|}
\hline Não & 348 & 96,9 & 587 & 96,7 & & & & & & \\
\hline Sim & 11 & 3,1 & 20 & 3,3 & 1,07 & $0,40-2,85$ & 0,893 & 1,33 & $0,54-3,29$ & 0,536 \\
\hline \multicolumn{11}{|l|}{ Óbito } \\
\hline Não & 364 & 99,5 & 616 & 99,3 & & & & & & \\
\hline Sim & 2 & 0,5 & 3 & 0,5 & 0,88 & $0,08-9,32$ & 0,917 & 1,99 & $0,20-19,70$ & 0,555 \\
\hline \multicolumn{11}{|c|}{ Displasia broncopulmonar } \\
\hline Não & 303 & 85,8 & 552 & 90,9 & & & & & & \\
\hline $\operatorname{Sim}$ & 50 & 14,2 & 55 & 9,1 & 0,66 & $0,37-1,19$ & 0,170 & 1,05 & $0,54-2,06$ & 0,879 \\
\hline \multicolumn{11}{|c|}{ Hemorragia intracraniana } \\
\hline Não & 206 & 78,9 & 267 & 78,1 & & & & & & \\
\hline $\operatorname{Sim}$ & 55 & 21,1 & 75 & 21,9 & 1,07 & $0,54-2,11$ & 0,841 & 1,56 & $0,71-3,42$ & 0,269 \\
\hline \multicolumn{11}{|l|}{ Apnéia } \\
\hline Não & 307 & 94,5 & 542 & 96,6 & & & & & & \\
\hline Sim & 18 & 5,5 & 19 & 3,4 & 0,61 & $0,23-1,61$ & 0,317 & 0,73 & $0,25-2,16$ & 0,573 \\
\hline \multicolumn{11}{|l|}{ Sepse } \\
\hline Não & 332 & 92,2 & 590 & 96,1 & & & & & & \\
\hline $\operatorname{Sim}$ & 28 & 7,8 & 24 & 3,9 & 0,50 & $0,17-1,46$ & 0,205 & 0,54 & $0,18-1,60$ & 0,264 \\
\hline \multicolumn{11}{|c|}{ Distermia } \\
\hline Não & 347 & 96,9 & 552 & 91,1 & & & & & & \\
\hline Sim & 11 & 3,1 & 54 & 8,9 & 2,88 & $0,75-11,04$ & 0,122 & 2,73 & $0,74-10,05$ & 0,131 \\
\hline \multicolumn{11}{|c|}{ Aleitamento exclusivo na alta } \\
\hline Não & 275 & 76,2 & 189 & 30,8 & & & & & & \\
\hline $\operatorname{Sim}$ & 86 & 23,8 & 425 & 69,2 & 2,89 & $1,43-5,83$ & 0,003 & 2,34 & $1,13-4,82$ & 0,022 \\
\hline
\end{tabular}

IC95\% = intervalo de confiança de 95\%; RR = risco relativo; UTI = unidade de terapia intensiva.

* Modelos ajustados para peso de nascimento, idade gestacional, SNAPPE II, NTISS, idade e escolaridade maternas e corrigidos pelo efeito cluster (unidade de nascimento). Os totais diferem para cada variável por causa de dados ignorados. 
para as mães. Isto pode ter reduzido ou anulado eventuais diferenças entre os dois grupos.

Houve perda de $15 \%$ no peso com 36 semanas de IG corrigida devido à alta da unidade intermediária convencional ou canguru antes desta IG. Perdas superiores a $20 \%$ também ocorreram para comprimento e PC com 36 semanas de IG corrigida. Estas medidas não foram obtidas diretamente pelas equipes de pesquisa e necessitaram da colaboração dos serviços e, apesar de todos os esforços despendidos, algumas perdas não puderam ser evitadas. Houve também perdas de $32,3 \%$ para retinopatia da prematuridade e de $38,8 \%$ para hemorragia intracraniana devido a dificuldades operacionais de realização de exames especializados de ultra-sonografia e fundo de olho.

O tamanho de amostra permitiu um bom poder de estudo para detectar diferenças nas médias das variáveis quantitativas, mas o poder foi mais baixo para detectar diferenças menores entre os percentuais de variáveis categóricas.

Outra dificuldade foi a comparação dos dados com o de outros trabalhos na literatura. A definição do chamado método canguru é extremamente heterogênea nos trabalhos consultados. Nenhum deles estabelece claramente a internação da mãe junto ao bebê nos cuidados intermediários como elemento imprescindível ao método, como estabelecido pelo Ministério da Saúde brasileiro. Além disso, o contato pele a pele é praticado segundo os mais variados protocolos. Além disso, enquanto alguns artigos utilizaram ensaio clínico randomizado, outros realizaram estudo observacional de coorte. Essas variações na definição do que seja o método canguru e no desenho do estudo podem explicar as diferenças observadas entre os estudos.

Desigualdades no uso de tecnologias entre as unidades podem explicar as diferenças em relação ao menor peso, comprimento e PC com 36 semanas de IG corrigida observados nas crianças das unidades canguru. Por outro lado, as unidades canguru tiveram desempenho superior em relação à promoção do aleitamento materno. O maior percentual de aleitamento exclusivo na alta não se deveu ao fato de os recém-nascidos do grupo canguru terem maior peso de nascimento, maior IG e serem menos graves ao nascimento, pois foi realizado ajuste para estes fatores.

Em conclusão, as evidências sugerem que a estratégia de humanização adotada pelo Ministério da Saúde é uma alternativa segura ao tratamento convencional e uma boa estratégia para a promoção do aleitamento materno.

\section{Agradecimentos}

A todas as maternidades e profissionais envolvidos na pesquisa e especialmente à coordenação e técnicos da área da criança e aleitamento materno do Ministério da Saúde. Também gostaríamos de agradecer especialmente a Fernando Barros e aos funcionários do Ministério da Saúde, desde o início envolvidos no projeto: Ana Cecília Sucupira, Aléxia Ferreira, Catarina Schubert.

\section{Referências}

1. Mathelin C. O sorriso da Gioconda: clínica psicanalítica com bebês prematuros. Rio de Janeiro: Companhia de Freud; 1999.

2. Lamy ZC. Unidade neonatal: um espaço de conflitos e negociações [Tese]. Rio de Janeiro, RJ: Fundação Oswaldo Cruz; 2000.

3. Walsh-Sukys M, Reitenbach A, Hudson-Barr D, DePompei P. Reducing light and sound in the neonatal intensive care unit: an evaluation of patient safety, staff satisfaction, and costs. J Perinatol. 2001;21:230-5. Erratum in: J Perinatol. 2001; 21:572.

4. Martín Ancel A, Iriondo Sanz M, Fina Martí A, Roqués Serradilla $V$, García Del Río M, López Sastre JB, et al. On recommendations, protocols, and clinical guidelines. An Esp Pediatr. 2001; 55:99-100.

5. Brasil. Ministério da Saúde. Norma da atenção humanizada ao recém-nascido de baixo peso - Método Canguru. Brasília, DF: MS; 1999.

6. Brasil. Ministério da Saúde. Norma da atenção humanizada ao recém-nascido de baixo peso - Método Canguru. Brasília, DF: MS; 2000.

7. Rey E, Martínez H. Manejo racional del niño prematuro. Bogotá, (Colombia): Universidad Nacional; Curso de Medicina Fetal; 1983.

8. Anderson GC, Marks EA, Wahlberg V. Kangaroo care for premature infants. Am J Nurs. 1986;86:807-9. Erratum in: Am J Nurs. $1986 ; 86: 1000$.

9. Anderson GC. Current knowledge about skin-to-skin (kangaroo) care for preterm infants. J Perinatol. 1991;11:216-26.

10. Conde-Agudelo A, Diaz-Rossello JL, Belizan JM. Mãe canguru para reduzir morbidade e mortalidade em recém-nascidos com baixo peso ao nascer (Cochrane Review). In: Resumos de Revisões Sistemáticas em Português, Issue 2, 2007. Oxford: Update Software.

11. Feldman R, Weller A, Sirota L, Eidelman AI. Skin-to-Skin contact (Kangaroo care) promotes self-regulation in premature infants: sleep-wake cyclicity, arousal modulation, and sustained exploration. Dev Psychol. 2002;38:194-207.

12. Kirsten GF, Bergman NJ, Hann KM. Kangaroo mother care in the nursery. Pediatr Clin North Am. 2001;48:443-52.

13. Ramanathan K, Paul VK, Deorari Ak, Taneja U, George G. Kangaroo Mother Care in very low birth weight infants. Indian J Pediatr. 2001;68:1019-23.

14. Ludington-Hoe SM, Johnson MW, Morgan K, Lewis T, Gutman J, Wilson PD, et al. Neurophysiologic assessment of neonatal sleep organization: preliminary results of a randomized, controlled trial of skin contact with preterm infants. Pediatrics. 2006; 117:e909-23.

15. Gray L, Watt L, Blass EM. Skin-to-skin contact is analgesic in healthy newborns. Pediatrics. 2000;105:e14.

16. Charpak N, Ruiz-Pelaez JG, Figueroa de CZ, Charpak Y. A randomized, controlled trial of kangaroo mother care: results of follow-up at 1 year of corrected age. Pediatrics. 2001; 108:1072-9.

17. Penalva O, Schwartzman JS. Descriptive study of the clinical and nutritional profile and follow-up of premature babies in a Kangaroo Mother Care Program. J Pediatr (Rio J). 2006;82:33-9. 
18. Charpak N, Ruiz-Pelaez JG, Figueroa de CZ, Charpak Y. Kangaroo mother versus traditional care for newborn infants $</=2,000$ grams: a randomized, controlled trial. Pediatrics.1997; 100:682-8.

19. Ruiz-Peláez JG, Charpak N, Cuervo LG. Kangaroo Mother Care, an example to follow from developing countries. BMJ. 2004; 329:1179-81.

20. Venancio SI, Almeida H. Método Mãe Canguru: aplicação no Brasil, evidências científicas e impacto sobre o aleitamento materno. J Pediatr (Rio J). 2004;80:S173-80.

21. Richardson DK, Corcoran JD, Escobar GJ, Lee SK. SNAP-II and SNAPPE-II: Simplified newborn illness severity and mortality risk scores. J Pediatr. 2001;138:92-100.

22. Gray JE, Richardson DK, McCormick MC, Workman-Daniels K, Goldmann DA. Neonatal therapeutic intervention scoring system: a therapy-based severity-of-illness index. Pediatrics. 1992;90:561-7.

23. Barros AJ, Hirakata VN. Alternatives for logistic regression in cross-sectional studies: an empirical comparison of models that directly estimate the prevalence ratio. BMC Med Res Methodol. 2003;3:21.

24. Roberts KL, Paynter C, McEwan B. A comparison of kangaroo mother care and conventional cuddling care. Neonatal Netw. 2000;19:31-5.
25. Cattaneo A, Davanzo R, Worku B, Surjono A, Echeverria M, Bedri $A$, et al. Kangaroo mother care for low birth weight infants: a randomised controlled trial in different settings. Acta Paediatr. 1998;87:976-85.

26. Kadam S, Binoy S, Kanbur W, Mondkar JA, Fernandez A. Feasibility of kangaroo mother care in Mumbai. Indian J Pediatr. $2005 ; 72: 35-8$.

27. Sloan NL, Leon Camacho LW, Pinto Rojas E, Stern C. Maternidad Isidro Ayora Study Team. Kangaroo mother method: randomised controlled trial of an alternative method of care for stabilised low-birthweight infants. Maternidad Isidro Ayora Study Team. Lancet. 1994;344:782-5.

Correspondência:

Fernando Lamy Filho

Hospital Universitário - Unidade Materno-Infantil/Serviço de Neonatologia

Rua Silva Jardim, 215 - 30 andar

CEP 65021-000 - São Luís, MA

Tel.: (98) 2109.1170

Fax: (98) 3248.2421

E-mail: lamyfilho@gmail.com 